\title{
At the molecular resolution with MINFLUX?
}

\author{
Kirti Prakash ${ }^{\mathrm{a}, \mathrm{b}, *}$ \\ ${ }^{a}$ National Physical Laboratory, TW11 0LW Teddington, UK; ${ }^{b}$ Department of Chemistry, University of Cambridge, CB2 1 EW Cambridge, \\ United Kingdom; * Correspondence: kirtiprakash2.71@gmail.com, Vkirtiprakash25
}

\begin{abstract}
Gwosch et al. (2020) and Balzarotti et al. (2017) purport MINFLUX as the next revolutionary fluorescence microscopy technique claiming a spatial resolution in the range of $1-3 \mathrm{~nm}$ in fixed and living cells. Though the claim of molecular resolution is attractive, I am concerned whether true $1 \mathrm{~nm}$ resolution has been attained. Here, I compare the performance with other super-resolution methods focusing particularly on spatial resolution claims, subjective filtering of localisations, detection vs labelling efficiency and the possible limitations when imaging biological samples containing densely labelled structures. I hope the analysis and evaluation parameters presented here are not only useful for future research directions for singlemolecule techniques but also microscope users, developers and core facility managers when deciding on an investment for the next 'state-of-the-art' instrument.
\end{abstract}

MINFLUX | STED | SMLM | localisation precision | image resolution | super-resolution imaging

\section{Introduction}

The spatial resolution of light microscopy is continuously being pushed with the development of new technologies. The significant milestones have been confocal laser scanning microscopy (Minsky, 1988), 2-photon microscopy (Denk et al., 1990), 4Pi microscopy (Hell et al., 1994), stimulated emission depletion (STED) microscopy (Hell and Wichmann, 1994), zero-point STED (Baer, 1999), structured illumination microscopy (SIM) (Heintzmann and Cremer, 1999; Gustafsson, 2000) and singlemolecule localisation microscopy (SMLM) (Lidke et al., 2005; Betzig et al., 2006). Recently, hybrid super-resolution microscopy techniques have further pushed the spatial resolution down to a few nanometers. These can be further divided into direct combinations of SMLM+STED (for scanning the position of the molecule with a donut) as in MINFLUX (Balzarotti et al., 2017) and SMLM+SIM as in SIMFLUX (Cnossen et al., 2020; Jouchet et al., 2019) or sequential correlative combinations of SIM and SMLM (Rossberger et al., 2013) and STED and SMLM (Prakash, 2021).

The major advantage of the direct combination is in resolution enhancement by increasing the information per photon. For readers interested in the technical details of these methods, following are some excellent recent reviews on this topic (Reymond et al., 2020; Schermelleh et al., 2019; Sahl et al., 2017; Heintzmann, 2021; Prakash et al., 2021). MINFLUX and SIMFLUX have demonstrated a resolution of less than 10 $\mathrm{nm}$ on synthetic structures like DNA origami and over $40 \mathrm{~nm}$ on biological structures. However, as most of the biologically relevant structures are novel and lie between 10-200 nm range, sequential correlative methods have a distinct advantage to independently validate new morphological findings using an orthogonal technique with a reasonably close resolution.

MINFLUX is presently the most photon efficient method to localize molecules and the aim of this article is not to argue otherwise. Here, I evaluate MINFLUX on the following five broad categories:

1. New biological insights through molecular resolution

2. Apriori structural information and subjective event filtering

3. Multi-colour, 3D, live imaging and the caveats when imaging ideal, well-defined structures

4. Spatial resolution vs localisation precision, density

I hope this detailed categorisation helps scientists to evaluate whether MINFLUX is the right microscopy technique for their research.

\section{New biological insights through molecular resolu- tion?}

The primary highlight of Gwosch et al. (2020) paper is the ability to resolve the individual components of nuclear pore complexes (NPCs) at the molecular scale (Figure 1, fourth row). NPCs have eight subunits, each with 4 copies of Nup96 and MINFLUX claims to have the resolution to resolve these 4 copies. Here, I re-examine this claim to probe if this molecular resolution provided new biological insights or was simply a visualisation enhancement scheme? 


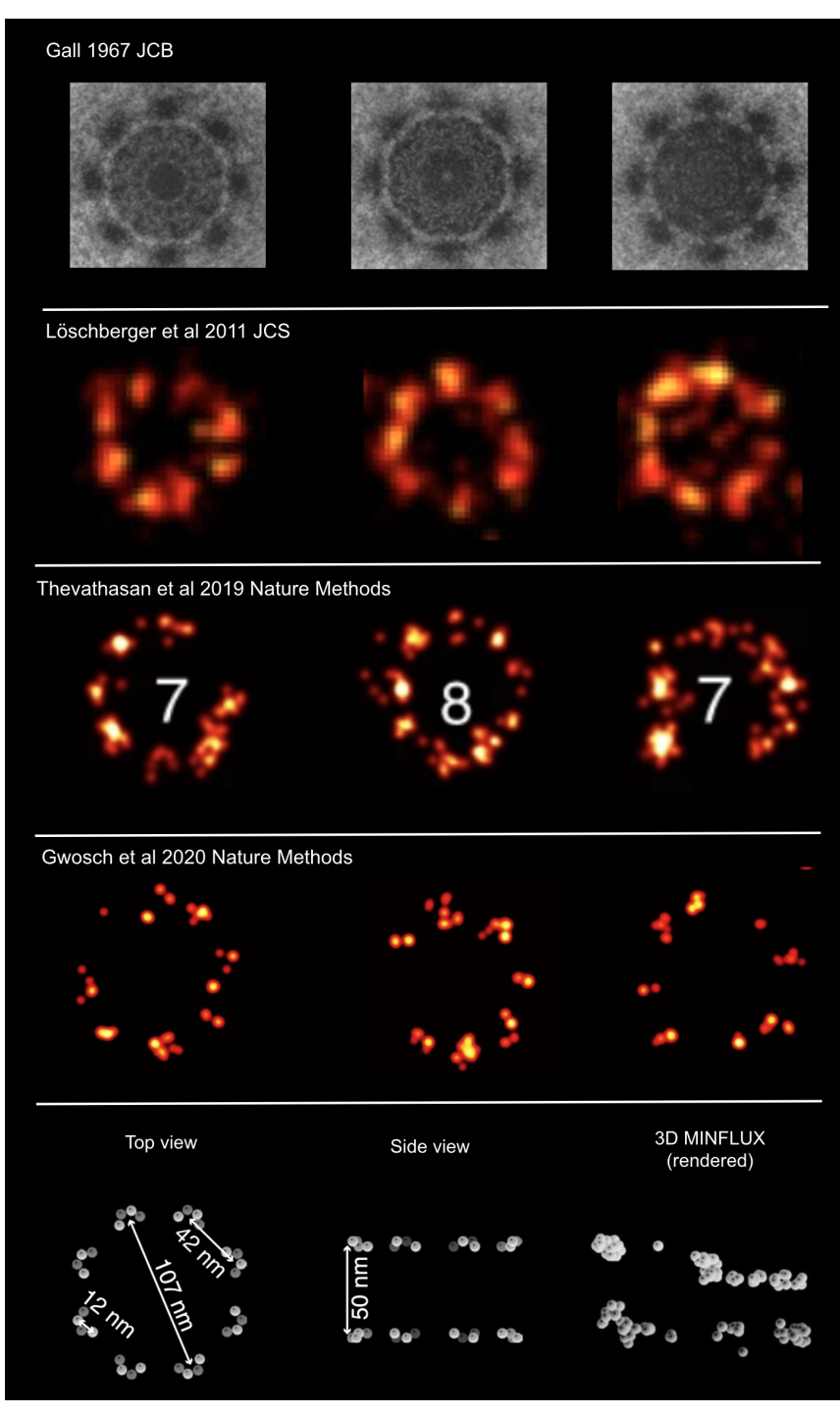

Figure 1. The need for molecular resolution? Nuclear pores across different imaging modalities:

(First row) Nuclear envelope from amphibian oocyte imaged with electron microscopy. The 8-fold symmetry of the nuclear pores is visible. The pore perimeter shows up as an octagon. Image adapted from Gall (1967).

(Second row) Membrane protein gp210 from amphibian oocyte imaged with dSTORM (Alexa Fluor 647). The 8-fold symmetry and the circular structures of NPCs is generally seen. The outer diameter is $\sim 120 \mathrm{~nm}$ and FWHM of gp210 is 30 nm. Image adapted from Löschberger et al. (2012).

(Third row) Nup96 endogenously labelled with SNAP-tag (Alexa Fluor 647 ) in U2OS cell lines. 8- and 7-component pores are more commonly observed. The effective labelling efficiencies for SNAP-Alexa Fluor 647 was $\sim 60 \%$. Image adapted from Thevathasan et al. (2019).

(Fourth row) MINFLUX imaging of U2OS cell expressing Nup96-SNAP labelled with Alexa Fluor 647. 6- and 7-component nuclear pores are more prominent raising questions on detection efficiency of the method. A special image rendering was used to visualise the 4 individual copies per subunit of NPCs, which appears as a blob for multi-color MINFLUX imaging see Figure 2. Cell line and labelling strategy same as in Thevathasan et al. (2019). Image adapted from Gwosch et al. (2020).

(Fifth row) A schematic of NPCs with the dimensions Nup-96, taken from Thevathasan et al. (2019). A 3D MINFLUX 'rendered' data presented for comparison from Gwosch et al. (2020). Colormap removed for a fair comparison. Notice the highly clustered, under-sampled and uneven distribution of well-defined periodic nuclear pores.

\section{A. New insights into nuclear pore biology: NPCs are symmet-} rical structures of eight subunits arranged in an octagonal
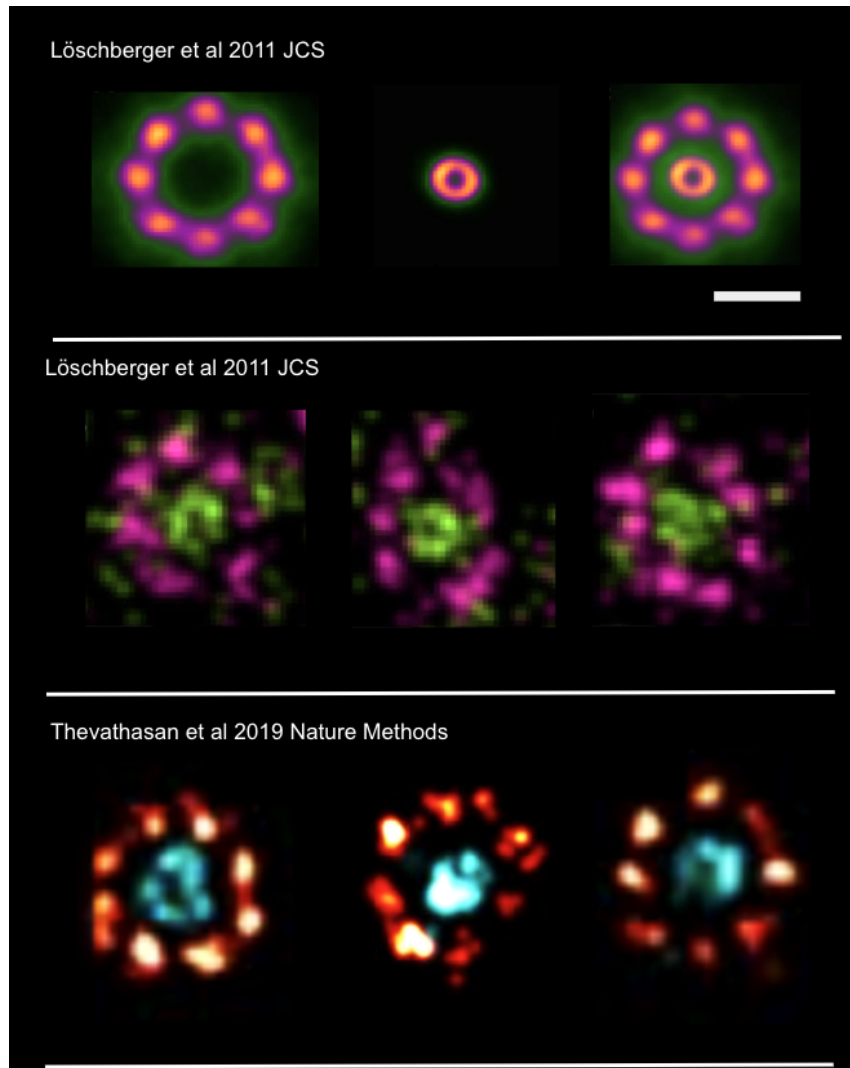

Gwosch et al 2020 Nature Methods

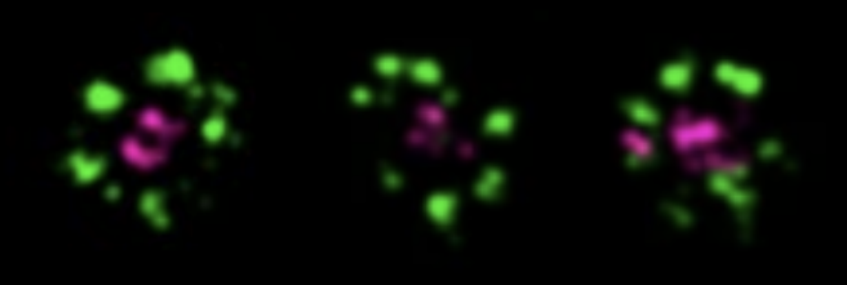

Figure 2. The missing molecular components with two-colour MINFLUX imaging:

(First row) Schematic for gp210 (the outer ring) and WGA (the central channel) of the NPC. The outer ring (gp210) has an average diameter of $\sim 120 \mathrm{~nm}$. The diameter of the inner ring (WGA) is $41 \pm 7 \mathrm{~nm}$ (true value $\sim 50 \mathrm{~nm}$ ). Image adapted from Löschberger et al. (2012). Scale bar: 100 $\mathrm{nm}$.

(Second row) dSTORM images of WGA labelled with ATTO 520 (green) and gp210 labelled with Alexa Fluor 647 (pink) in amphibian oocytes. Both the outer ring and inner channel are visible (Löschberger et al., 2012).

(Third row) Two-color SMLM image of Nup96-SNAP labelled with Alexa Fluor 647 (red) and WGA-CF680 (cyan) in U2OS cell lines. The outer ring is clearly visible and the inner ring is also visible in most cases. Image adapted from Thevathasan et al. (2019).

(Fourth row) Two-color MINFLUX imaging of U2OS cell expressing Nup96-SNAP labelled with Alexa Fluor 647 and WGA conjugated to CF680. The outer ring where each of 8 NPC subunits have 4 copies (as in Figure 1), now appear as a blob. The inner ring (WGA) also aggregates as blobs. Image adapted from Gwosch et al. (2020).

It must be noted that the inner and outer of nuclear pores in Thevathasan et al. (2019) and Gwosch et al. (2020) were imaged in 3D, while in Löschberger et al. (2012) were in 2D.

geometry with an outer diameter of approximately $120 \mathrm{~nm}$ (see Figure 1, fifth row for a detailed schematic). There are 32 copies of Nup96 per NPC with each of the 8 subunits having 4 copies. These 4 copies are roughly around $12 \mathrm{~nm}$ in diameter. 
The organisation of the 4 copies in the individual subunits is still unknown. With a 1-3 $\mathrm{nm}$ resolution range of MINFLUX, a general expectation is to gain structural insights into the organisation of individual components of the NPCs. Do the 4 copies have some ordered organisation (for example, a tetrahedron) or are they randomly distributed? As no observation on the internal organisation of these copies (12 nm structures) are made, it raises questions on the actual usefulness of 1-nm resolution of MINFLUX to provide new structural insights.

B. Need for independent validation: It is worth noting that no independent validation of molecular copies or subunits of NPCs was done in Gwosch et al. (2020) using either STED or SMLM. Moreover, Thevathasan et al. (2019) used the same cell line (U2OS) and labelling strategy (Nup96-SNAP) as in Gwosch et al. (2020) (same authors) and provides an easy basis for direct comparison. The molecular components are 12 $\mathrm{nm}$ in diameter and $42 \mathrm{~nm}$ apart, so should be easily resolved by both MINFLUX and SMLM.

As far as the organisation of 8 subunits of NPCs is concerned, they have been resolved with electron microscopy (EM) and single-molecule localisation microscopy (SMLM) roughly 50 and 10 years ago, respectively (Gall, 1967; Löschberger et al., 2012). For a comparison of NPCs using EM and SMLM see Figure 1.

C. Detection vs labelling efficiency: Gwosch et al. (2020) used Nup96 endogenously labelled with SNAP-tag (Alexa Fluor 647) in U2OS cell lines, same as in Thevathasan et al. (2019). As a general observation, 2-3 components per pore are often missing in MINFLUX data (Figure 1, fourth row). MINFLUX images show NPCs with 6- and 7-components, which is not the case with SMLM data. This raises concerns on the detection efficiency of MINFLUX and role of event filtering (see next section). If the missing components are due to labelling efficiency of SNAP-tag then this would be observed in the SMLM data of Thevathasan et al. (2019). Moreover, if the lack of labelling would be random, 1-2 copies of Nup96 would be missing per subunit of the pore, reducing the intensity but not the entire NPC subunit. It is very likely that the subjective filtering of localisations based on photon counts and the distance from the estimated position (structure assumption) leads to the missing of the pore subunit.

D. MINFLUX and the case of fewer photons: Gwosch et al. (2020) state that MINFLUX requires fewer detected photons when compared to camera-based localization methods like PALM/STORM. So it is applicable to a large range of fluorophores and labelling strategies and is "bound to be a cornerstone, if not the vanguard, of nanometer-scale fluorescence."
However, as of now, MINFLUX has made use of one of the brightest and the best fluorophores for localisation microscopy namely Alexa Fluor 647 and has not done any systematic comparison between dyes or different super-resolution methods. In Balzarotti et al. (2017) MINFLUX comparison with PALM/STORM (using DNA origami) was only a simulation and no experimental data was presented. It is worth noting that the authors as part of another paper (Thevathasan et al., 2019) created four cell lines for Nup96 which also includes mEGFP. mEGFP and other organic dyes have poor emission stability and as MINFLUX localisation precision lies on successive measurements of intensities over each ON cycle, it will be beneficial for community to see performance of these dyes. For a relative comparison of SMLM and MINFLUX using taxane analogs coupled to red fluorescent dyes (HMSiR) (Gerasimaite et al., 2021), please see Figure 3.

\section{Apriori structural information and event filtering}

For years, in electron and single-molecule localisation microscopy, filtering of imaging data has been done to enhance contrast and optimise visualisation. In this section, I highlight how event filtering can be used to attain higher localisation precision, purport new biological structures not present in the raw data and the need for blind samples to standardise the resolution claims.

A. Localisation filtering at manually defined positions: To highlight the role of localisation filtering and apriori information to achieve molecular resolution, I use nuclear pore data from (Thevathasan et al., 2019), available at https://www.ebi.ac.uk/biostudies/Biolmages/studies/S-BIAD8. For downstream analysis at individual nuclear pore level, I zoomedin at an image section with 4831 localisations (Figure 4). For these localisations, the observed bimodal precision plot (centre at $22 \mathrm{~nm}$ ) was used for further filtering of localisations. The same nuclear pore section with different filtering of localisations at a manually chosen threshold is shown in the bottom row, all columns (Figure 4).

A single nuclear pore (yellow box) is further highlighted to show the impact of visualisation and image rendering. Notice how the individual components of the 8 subunits of NPCs cannot be resolved with image zoom but can be resolved with a manually chosen pixel size $(0.5 \mathrm{~nm})$ and Gaussian kernel (8 $\mathrm{nm}$ ). The choice of pixel size and Gaussian kernels were done with a prior understanding of how the NPCs 'should' look like. The filtering threshold $(5 \mathrm{~nm})$ was chosen to provide a close to 4 copies per subunits. In this regard, we question the choice of pixel and kernel size used in Gwosch et al. (2020). For example, a pixel size of $0.5 \mathrm{~nm}$ and a large-width Gaussian kernel of $4 \mathrm{~nm}$ was used for Figure $2 \mathrm{a}$ and $5 \mathrm{e}$ but a kernel of 2 
(a)

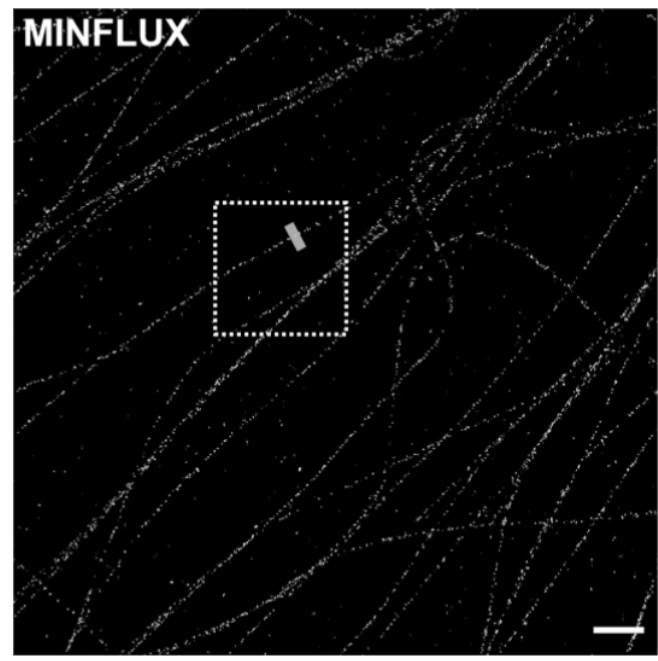

(b)

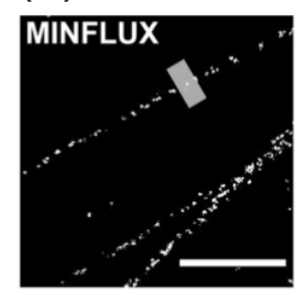

(c)

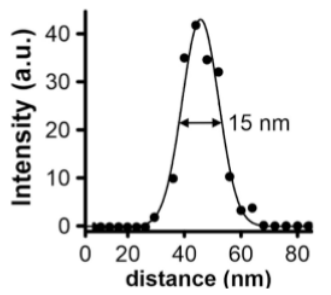

(d)

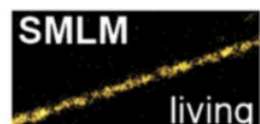

SMLM

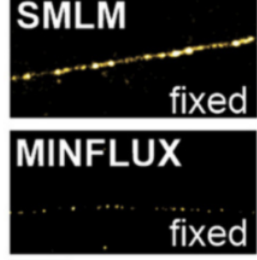

$\overline{500 \mathrm{~nm}}$ (e)

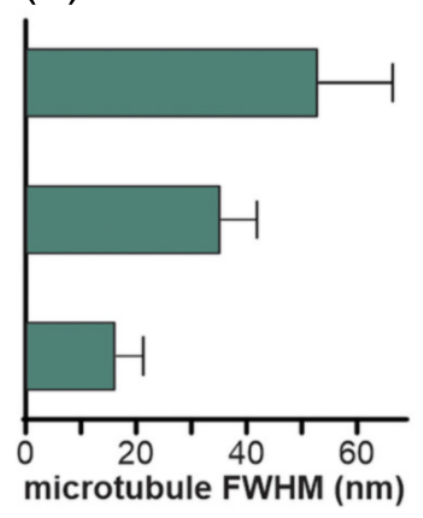

Figure 3. Localisation precision Vs localisation density: (a, b) MINFLUX imaging of microtubules using HMSiR probe. (c) FWHM of inner diameter of microtubule. The standard estimate for inner diameter of microtubule is $17 \mathrm{~nm}$ ( $25 \mathrm{~nm}$ for the outer diameter diameter). (d) A comparison of SMLM and MINFLUX using the same HMSiR probe. (e) The corresponding FWHM of microtubules (live, fixed for SMLM and fixed for MINFLUX). Though MINFLUX achieves better precision than SMLM, the localisation probability and density remains poor. One of the overlooked parameters in single-molecule imaging is sampling and the requirement to have a minimum number of localisation to decipher the underlying structure. Here, a prior knowledge of the hollow cylindrical structure of microtubule is assumed to map the dots-on-line configuration to the under-sampled data. The successive measurement of intensities especially of dyes with poor emission stability is also likely to have an impact on the sampling and localisation density. Figure was kindly provided by Gražvydas Lukinavičius and data from Gerasimaite et al. (2021).

$\mathrm{nm}$ and a pixel size of $0.2 \mathrm{~nm}$ for Figure $2 \mathrm{a}$ and $2 \mathrm{f}$ in Gwosch et al. (2020).

B. The filtered MIFNLUX data: The MINFLUX filtering is done at various levels based on photon counts, targeted coordinate pattern to account for true emission and those considered as background events. For a fair comparison, both raw and filtered data should be presented to demonstrate that any such filtering is not biased by apriori structural information. For structures with prior information like NPCs, the manual filtering can lead to additional biases for live imaging, see Figure 6B. For structures with little prior information, the reduction in the number of molecules would lead to under-sampled structure (Figure 6A). Furthermore, it raises questions on the authenticity of the structures if an independent validation via electron microscopy or other super-resolution methods is not provided. Lastly, it is not clear if the molecular components of NPCs will still be visible in raw/unfiltered data as the increased density of signals will tend to overlap like in the two-colour images (Figure 2). Worth noting that subjective manual filtering with better localised molecules (as in Figure S4 of Gwosch et al. (2020)) leads to an increased resolution estimate (see Figure 5 for a comparison).

C. DNA origami and the need for blind samples: DNAorigami is commonly used to measure the spatial resolution for different microscope setups. DNA origami has a pre-defined arrangement and blind samples with no knowledge on the prior arrangement are needed for proper calibration. This will make the photon count based filtering more accountable as origami samples, unlike biological samples, have almost zero background (see Figure 5). As signal-to-noise ratio is arguably the most important aspect in biological imaging, the effectively achieved resolution is heavily dependent on it and and can vary across sample regions. It is important to understand that unlike an implied single maximum resolution value, the effective resolution is variable, in particular due to the fluctuating contribution of light scatter and out-of-focus blur in different sample regions within the field of view.

Results obtained by imaging and analysing DNA origami will likely be a poor predictor of performance for real biological samples where problems of out-of-focus blur, non-specific background, light scattering and other sample aberrations exist. Thus, the resolution claimed based on DNA origami needs to be thoroughly investigated in this context and efforts made not to mislead the researchers interested in real biology.

In fact, a new quality metrics for microscopy techniques of the relative deviation between nominal and real life resolution in different biological situations needs to be introduced. After all, conventional fluorescence microscopy rarely achieves its theoretical limit. However, the relative deviation is much smaller than in MINFLUX. 


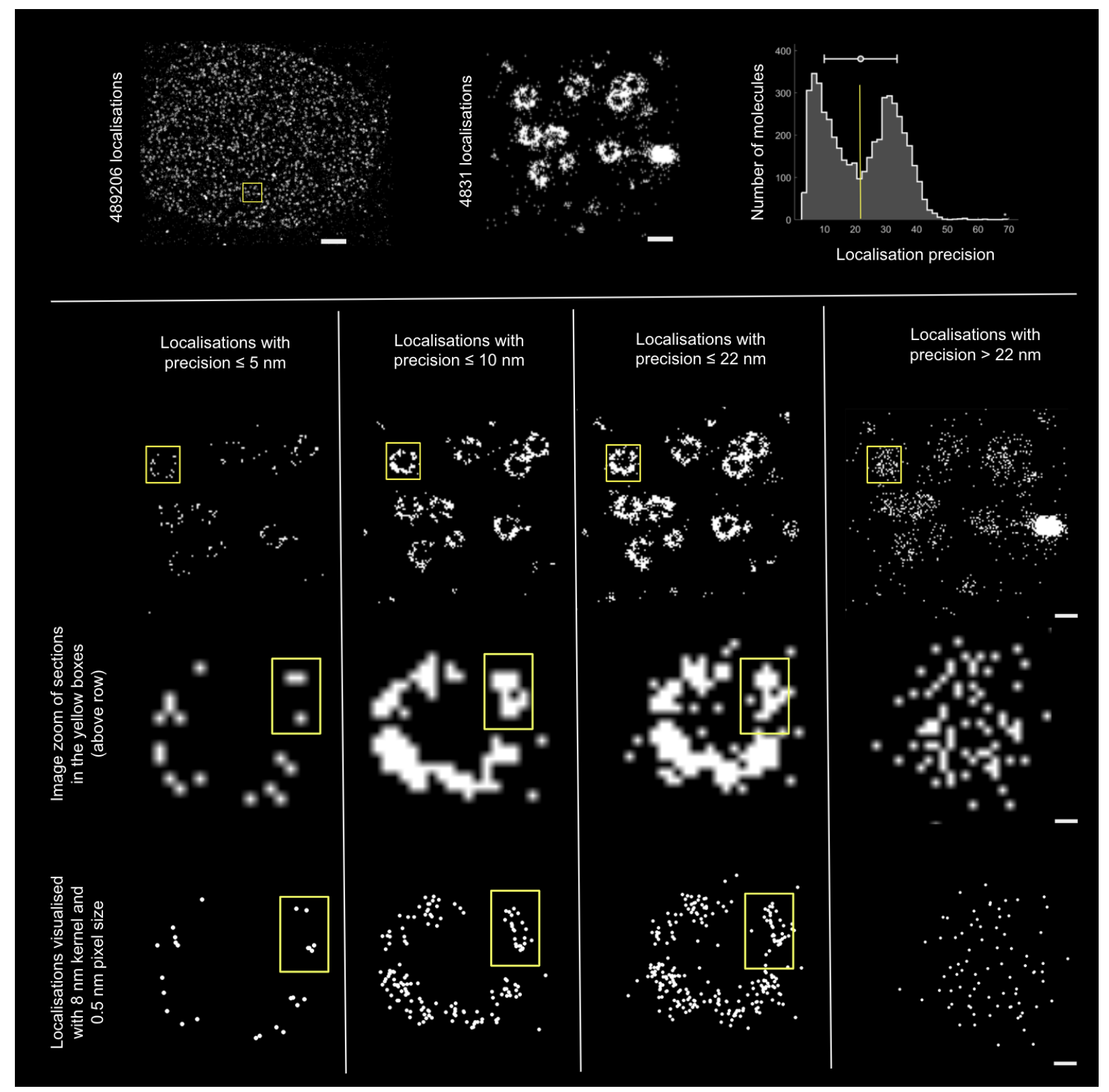

Figure 4. Filtering of localisations, visualisation enhancement and molecular resolution:

(Top row) (Left) Nuclear pore complexes (NPCs) data from Thevathasan et al. (2019), accessed from https://www.ebi.ac.uk/biostudies/Biolmages/studies/ S-BIAD8. Scale bar: $1000 \mathrm{~nm}$. (Middle) A zoomed-in excerpt (yellow box) with 4831 localisations for further downstream analysis in the panels below. Scale bar: $100 \mathrm{~nm}$. (Right) The localisation precision plot for the zoomed-in excerpt, notice the unexplained bimodal distribution.

(Bottom row, all columns) The same nuclear pore section with different filtering of localisations at a manually chosen threshold (Scale bar: $100 \mathrm{~nm}$ ). The zoomed-in excerpts of individual pores (yellow box) highlight the impact of visualisation and image rendering (Scale bar: $10 \mathrm{~nm}$ ). Notice how the individual components of the 8 subunits of NPCs which can not be resolved with Gaussian blurring, can now be resolved with a manually chosen pixel size and Gaussian kernel (bottom row). The choice of pixel size, Gaussian kernels were done with a prior understanding of how the NPCs 'should' look like. The filtering threshold for localization precision was chosen to provide a close to 4 copies per subunits. Often, in such cases, the missing copies are attributed to the limited efficiency of the labelling method.

\section{The choice of biological sample for resolution claims: For} the lateral spatial resolution, so far, MINFLUX imaging has been done on NPCs (Gwosch et al., 2020), microtubules (Gerasimaite et al., 2021), mitochondrial MICOS proteins (Pape et al., 2020) and post-synaptic protein PSD-95 (Gwosch et al., 2020). These can be classified as samples having a well-defined underlying structures (NPCs, microtubules) or with no inherent underlying structures (MICOS, PSD-95).

For the biological targets with no underlying structure, an independent validation with another super-resolution method or a relative comparison under identical imaging conditions and length scales is essential. For such studies, a minimum 

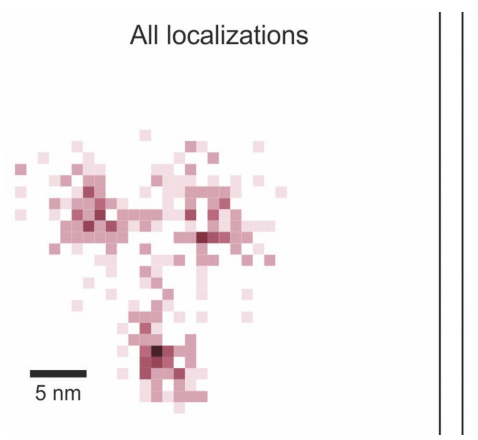

Localizations with $\mathrm{N}>1200$

Figure 5. MINFLUX WYAKIWYG: what you already know is what you get:

To demonstrate the difference between the experimental precision and the precision of the filtered data, I use p-MINFLUX data (Masullo et al., 2020) provided by Florian Steiner.

Masullo et al. (2020) state that to achieve a certain precision ( $2 \mathrm{~nm}$ ), a minimum number of photons $(>1200)$ are needed and is the primary reason for the choice of filtering threshold. For most biological samples, the underlying structure is almost always unknown, unlike here where the arrangement of DNA origami in 3-dots is known in advance. This can lead to subjective filtering thresholds for higher spatial resolution claims as long as 3-dots can still be distinguished. What if the arrangement of DNA origami was a T or Y shape, would the same filtering with events $>1200$ photons be still done?

I recommend use of blind samples with no knowledge about the prior arrangement of DNA origami for proper resolution calibration.

Note that the average precision of all the localizations would be much higher and this number has not been reported by the authors. The precision of all localisations is the true precision of the instrument and not the one calculated on the filtered data.

signal density and labelling efficiency should be considered as a minimum requirement.

For the biological targets with an underlying organisation such like NPCs, the filtering of data can be misused to attain a certain resolution (see Figure 5). Furthermore, such filtering would lead to biases in labelling and detection efficiency.

\section{Multi-colour, 3D, live MINFLUX imaging and caveats when imaging continuous well-defined structures}

MINFLUX was originally published in 2017 (Balzarotti et al., 2017) with nanometer resolution claim on a synthetic structure (DNA origami) and particle tracking in bacterial samples. In Gwosch et al. (2020), the authors, for the first time, claim nanometer resolution in biological samples, in three dimensions, in living cells and using multiple color channels. In this section, I evaluate method's potential and the strength of the reported data.

A. The missing molecular components in multi-color imaging: The individual proteins of the eight NPC subunits (roughly $40 \mathrm{~nm}$ apart) that can be observed in single-colour images, vanish in two-colour images (Figure 2) questioning the performance and resolution claim of MINFLUX under these imaging conditions. The molecularly resolved 4 copies of the subunits now appear as a 'blob' in 2-colour images. This also includes the inner ring of the WGA displayed as a blob in the second colour channel. Note that both the inner and outer ring are clearly visible in Löschberger et al. (2012) and Thevathasan et al. (2019) respectively. In summary, for 2 colour imaging experiments, MINFLUX appears to offer an effective lateral resolution that is no better than localisationbased super-resolution methods (see Figure 2 for a detailed comparison). Can the molecular resolution still be claimed given that no 'molecules' are observed in a 2-colour MINFLUX imaging experiment?

B. The rendered 3D MINFLUX data: Regarding the 3D imaging of well-defined structures like NPCs, the rendered MINFLUX data appears to be highly clustered, under-sampled and unevenly distributed (see Figure 1, fifth row for a comparison with schematic of 3D organisation of NPCs). Several SMLM papers have put out a high-quality 3D structure of NPCs and is difficult to compare them with the rendered data from Gwosch et al. (2020). The under-sampled data again raises questions on detection efficiency of MINFLUX and its applicability for nuclear pore biology. It is also noted that no quantitative comparison of MINFLUX for Z resolution with SMLM is provided. For excellent 3D nuclear pore data, please refer to Figure 1 of Thevathasan et al. (2019).

The two-color 3D images of the nuclear pores with WGA (CF680) and Nup96 (SNAP-Alexa Fluor 647) again are barely comparable with standard 3D SMLM images. The ring distribution of WGA is unresolved and instead appears like a random distribution of points. The Nup96 octamer is also hardly visible, both laterally and axially (see Figure 5 and Supplementary Video 3 of Gwosch et al. (2020)).

\section{MINFLUX imaging of continuous well-defined structures:} MINFLUX has been used to probe the sub-mitochondrial localization of the core MICOS proteins (Pape et al., 2020). The authors claim to observe ring-like arrangements with a diameter of 40 to $50 \mathrm{~nm}$. Upon close examination, it appears that ring-like arrangement is rather subjective and the readers are encouraged to interpret it themselves, see Figure 6a (this paper) or Figure 3A and Movie S6 of Pape et al. (2020). As no independent cross-validation of these ring-like strictures via electron microscopy or other super-resolution methods is provided, it raises concerns on the validity of the observed punctuated structures. These punctuation, at best, can be described as a highly under-sampled ring. At this point, it is not clear if this is due to the detection efficiency of MINFLUX and its limitation to image dense as well as continuous structures. This, in turn, highlights the need for sequential correlative super-resolution methods where new structural findings from one method can be independently validated under similar imaging conditions. Lastly, the claimed localisation precision 
(a)

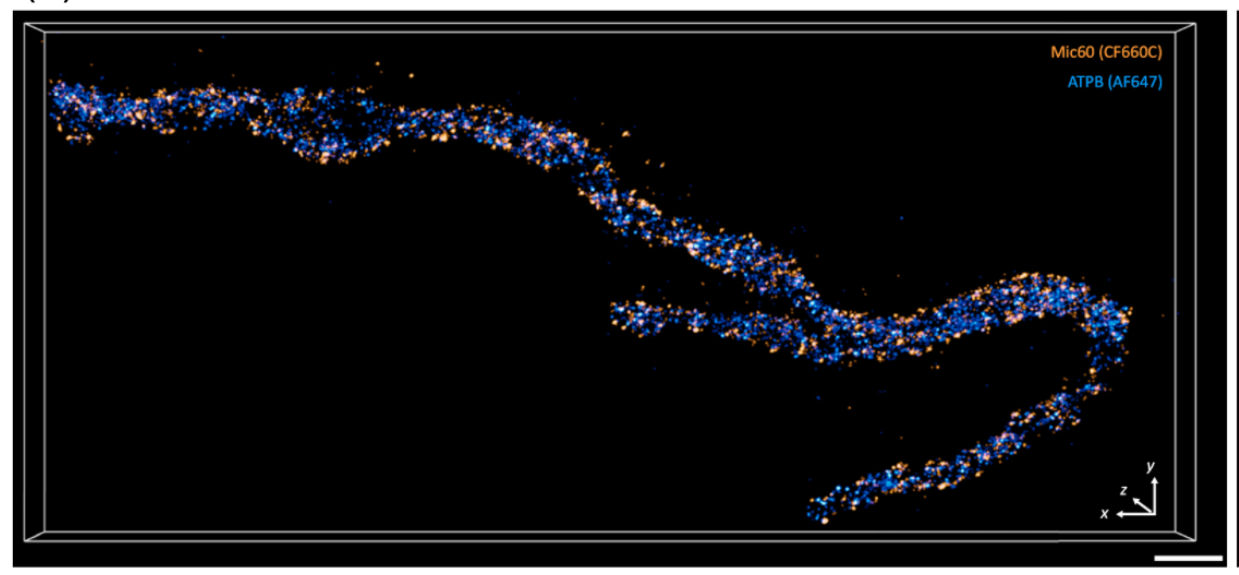

(b)

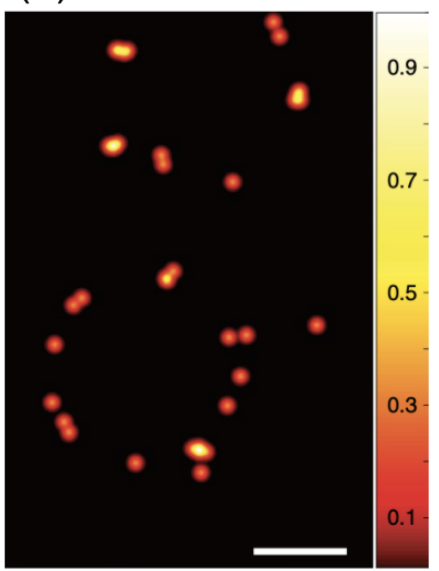

Figure 6. MINFLUX imaging of densely filled ring-like structures of Mic60 and Nup96:

(a) Pape et al. (2020) claim to observe ring-like arrangements of Mic60 molecules with a diameter of 40 to $50 \mathrm{~nm}$. The ring-like arrangement is rather subjective here and is left for the readers to interpret themselves (see Movie for more insights). As no independent validation of these ring-like strictures via electron microscopy or other super-resolution methods is provided in the paper, the observed punctuated structures can, at best, be considered as a highly under-sampled ring. This raises questions on the detection efficiency of the method and its ability to image dense as well as continuous structures Scale bar: $500 \mathrm{~nm}$

(b) Live MINFLUX imaging of nuclear pores expressing Nup96-mMaple in U2OS cell. Notice the highly under-sampled configuration of nuclear pores as only the localizations with more than 1600 photons were considered. Image adapted from Gwosch et al. (2020). Scale bar: $50 \mathrm{~nm}$.

of $5 \mathrm{~nm}$ with a structural resolution of 40-50 nm brings in the debate of the difference between experimental 'localisation precision' versus achieved practical 'structural resolution' and how these numbers should be reported.

The microtubules: Historically, FWHM of microtubules (MTs) has been the benchmark for resolution demonstration in STED microscopy. MTs are continuous and have a denser tubular organisation $(25 \mathrm{~nm})$ when compared to the nuclear pores. Hence, any subjective filtering would lead to an undersampled image. The hollow tube structure of MTs has been resolved with SMLM and DNA-PAINT setups. For a well resolved 3D cylinders of microtubules with SMLM, see Huang et al. (2016); Li et al. (2018). On the other hand, MINFLUX fails to achieve the same level of structural resolution, see Figure 3. Gerasimaite et al. (2021) claims $15 \mathrm{~nm}$ for the inner diameter of microtubule, whose interpretation is left to the readers (standard estimate of inner diameter is $25 \mathrm{~nm}$ ).

In summary, MINFLUX performance on continuous structures with a well-defined organisation, such as MTs or those for which prior information is not available, remains to be proven. For MICOS, post-synaptic proteins, etc., which do not have an underlying structure, any resolution and shape can be claimed but is it valid? Thick samples where background is high will be another big challenger for MINFLUX especially when light-sheet camera-based SMLM setups.

D. Live MINFLUX imaging: MINFLUX imaging of nuclear pores (Nup96-mMaple) in living U2OS cells shows a highly under-sampled configuration with no data on cell viability or photo-bleaching (Figure 6B). The observed uneven distribution of nuclear pores is possibly due to heavy filtering of localizations (>1600 photons), see Prakash and Curd (2021) for a detailed comparison.

Regarding excitation powers for live imaging, the authors used powers $\sim 20-60 \mu \mathrm{W}$ with the peak intensities of the donut beam $\sim 10-50 \mathrm{~kW} / \mathrm{cm}^{2}$. For single molecule techniques, the excitation power is $\sim 0.1 \mathrm{~kW} / \mathrm{cm}^{2}$ to switch the fluorophores between dark and bright state, $\sim 1000$-fold less (Betzig et al., 2006). For structured illumination and confocal microscopes, especially the laser-free versions, the excitation powers are in tens of $\mathrm{mW} / \mathrm{cm}^{2}$ range or below. Thus, for live imaging MINFLUX is still off by a significant margin with respect to excitation powers when compared to single-molecule imaging, structured illumination or confocal microscopy.

\section{Spatial resolution and localisation precision}

MINFLUX nanoscopy claims to provide a "resolution in the range of 1 to $3 \mathrm{~nm}$ for structures in fixed and living cells". It is important to remember that $1-3 \mathrm{~nm}$ range here refers to localisation precision, and more specifically of fluorophore and not that of the target protein (Figure 7). As argued in this paper, MINFLUX is yet to resolve structures below 40 $\mathrm{nm}$ in biological samples. For synthetic structures like DNA origami, subjective event filtering can lead to unrealistically high precision numbers.

A. Precision is not resolution: For years, localisation precision and spatial resolution have been interchangeably used by 
physicists given their inherent dependence on photons. Resolution i.e. the ability to resolve close-by structures or target molecules, primarily depends on how densely a biological structure is labelled while localisation precision implies how well these labels are detected, see Figure 7 for more details.

For single-molecule studies, localisation precision follows an inverse square-root relationship with the total number of detected photons, whereas MINFLUX has an inverse quadratic relationship with detected photons under ideal conditions with zero background. For real-life biological applications, MINFLUX is severely limited by the background photons as this compromises the positioning of the donut zero. In general, MINFLUX is likely to achieve better precision numbers than single-molecule imaging but so far, due to multiple reasons stated in this article, the spatial resolution of the method has been much poorer than SMLM methods (see Figure 1).

B. Resolution quantification: Gwosch et al. (2020) applied three different criteria for resolution quantification. The first approach was to calculate the standard deviation for $>5$ localizations/fluorophore, where each localization had $\sim 2000$ photons. The events with high photon counts would automatically result in higher precision numbers. Notice how Gwosch et al. (2020) begin with resolution quantification but quote the precision of filtered data, skipping the average precision of all localisations. This leads to confusing localisation precision of a fluorophore with the spatial resolution of the method.

The second resolution assessment is by subtracting the mean localized position from all localizations. This process is similar to the first approach and will be biased by the filtering of the data. The third approach is based on Fourier ring correlation (FRC) and it is not clear whether filtered or raw data was used for FRC analysis. FRC measures correlation between subset of localisations and will again by affected by filtering of the data.

Prakash and Curd (2021) estimated localization precision $\left(\sigma_{x y}\right)$ were $0.98 \pm 0.02 \mathrm{~nm}, 3.20 \pm 0.05 \mathrm{~nm}$ and $3.31 \pm 0.08 \mathrm{~nm}$ for the 2D, 3D 1-color and 3D 2-color MINFLUX datasets (Gwosch et al., 2020), respectively. FWHM $\approx 2.355 \sigma$, so $\sigma_{x y}$ of 1-3 nm implies FWHM of 2.4-7.1 nm for a single molecule. So, one would not expect to clearly resolve molecules closer than this.

C. 1-nm resolution and 3-nm linker: A resolution cannot be better than the localisation precision of the detected fluorophore. As a general rule, a more conservative upper limit for localisation precision or full-width at half-maximum should be used to account for the linkage errors. From a biochemistry point of view, how reasonable to have a resolution in the range of $1-3 \mathrm{~nm}$ when the SNAP-tag itself is about $3-3.5 \mathrm{~nm}$

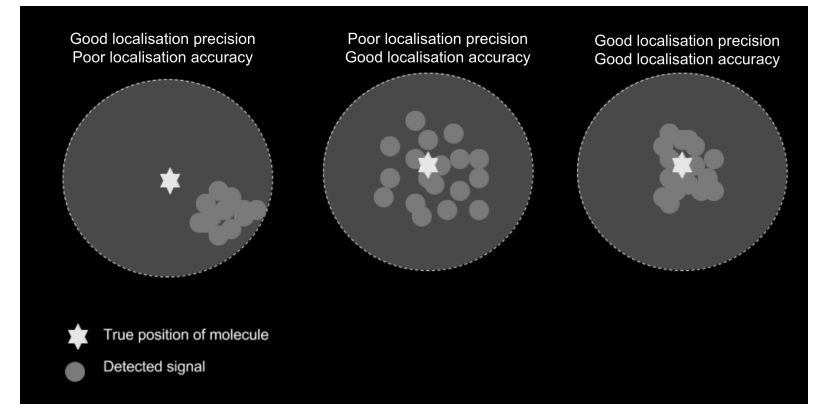

Figure 7. Localisation precision of the fluorophore or the protein of interest: The readers of super-resolution microscopy papers should look out when localisation precision is implied for spatial resolution. Resolution primarily depends on how densely a biological sample is labelled while localisation precision implies how well these labels are detected. Image adapted from Prakash (2017).

in size? Given the resulting uncertainty of relative position of the dye molecule, the stated nanometer resolution (or the localisation precision for that matter) can only be claimed for the fluorophore, but not the biological target. In this context, I urge the readers to take a careful note when authors state the precision of the fluorophore and that of the target protein.

D. Is it all about the principle and not practice?: Generally speaking, the resolution is the fundamental ability to determine a structure. From a physicist perspective it is satisfying that MINFLUX can achieve 1-nm localisation precision under ideal conditions (based on photon statistics), whether it materialises in resolving real life biology or not. From a biologist perspective, for a 1-nm resolution claim, MINFLUX is off by a factor of 5 for synthetic samples like DNA origami and a factor of 40 for biological samples like NPCs.

For a true 10-nm strucutral resolution, a general expectation would be to resolve a well-established $10 \mathrm{~nm}$ bead-on-string structure of nucleosomes or a turn of DNA helix which is about $3.4 \mathrm{~nm}$ (for 1-nm claim). From Nyquist viewpoint to achieve $10 \mathrm{~nm}$ structural resolution, one would need a dye every $4 \mathrm{~nm}$, which for many biological structures is hard to achieve due to steric hindrance or low copy number/binding sites. Alternatively, for structural biology, with true 1-nm resolution claim, I wonder if one can compare MINFLUX with $\mathrm{X}$-ray crystallography, which has a broad range of resolution (anything between 1.5 and $10 \AA$ ) depending on the crystal quality.

\section{Summary and the lessons from the past}

A. Towards standard biological reference structures: The spatial resolution in light microscopy has become something of a numbers game where scientists cite an arbitrary FWHM or an FRC resolution or filter localisations to achieve any desired resolution. Given the large number of potential biological 
reference structures in the range of 1-100 $\mathrm{nm}$ size scale, for example, DNA (2 nm), nucleosomes (10 nm), microtubules (20 $\mathrm{nm})$, NPCs (40 nm), synaptonemal complexes (60-150 nm), I recommend using these well-defined structures to support spatial resolution claims. For DNA origami researchers, I encourage usage of blind samples where no one knows the origami arrangement or the distances in advance. This will make the photon count based filtering more accountable.

B. Potential research areas for MINFLUX: Gwosch et al. (2020) and Balzarotti et al. (2017) have made efforts to push the localisation precision to single $\mathrm{nm}$ range which works well on ideal synthetic samples, but major compromises need to be made when applying these techniques to real-life biology. MINFLUX has value for a certain specific application (e.g. single-molecule tracking, material science i.e. nitrogen-vacancy centres), but in its current state, it should not be considered as the next-generation versatile imaging platform. Technical issues with detection, limitation when imaging for well-defined, continuous and dense structures, the use of well-characterised single-molecule dyes still limits its broader applicability.

C. Reproduction crisis: MINFLUX papers have shown several loop-holes in current scientific reporting and standards. A clear distinction needs to be made about experimental precision, spatial resolution, cross-validation of new structures by independent methods, extent of sample variability vs measurement uncertainty, hardware adaptability, image quality/standards (for example, acceptable measures for image rendering), availability of raw data/codes, reproducibility of the analysis pipeline, automated vs manual components of data analysis (like filtering) and proper procedures to report them (Hohlbein et al., 2021).

From a historical perspective, 4Pi, STED and now MINFLUX have been closed systems with little focus on making the hardware adaptable or raw data/codes available. Several units of highly-priced 4Pi microscopes were sold around 2000s as the next generation microscope, however, not many biological findings resulted. This was followed with the launch of STED microscopes and very few novel biological results.

Science is about reproducible measurements. Improvement in precision leads to refined measurements. MINFLUX improves the precision with which fluorophores can be localised but by not making the hardware adaptable, raw data and codes available, it is hindering reproducibility, open science efforts and overall progress of the field.

D. Important conceptual advancement vs current technical limitations: One of the important aspects of science is to promote cutting-edge ideas even if they offer little gains in the short run. MINFLUX research provides an important conceptual advancement and leads to overall progress in the field. The aim of this article is not to discourage MINFLUX research but to highlight that the technology is still underdeveloped and needs further validation from other independent labs before it is made commercially available especially considering the high price tag of the commercial system.

As research grants mostly come from tight public funds and are a zero-sum game, I hope this critique provides the scientists, microscope users, developers and core facility managers with an alternative viewpoint when deciding about an investment in the next "state-of-the-art" microscope instrument.

\section{Acknowledgements}

I thank Eric Betzig, Johannes Hohlbein, Lothar Schermelleh, Michael J Shaw, Richard W Clarke, Tulika Gupta and Rainer Heintzmann for useful discussion and/or comments on the manuscript. I further thank Alessandro Esposito, Jelmer Cnossen, Alex Merz, Francisco Balzarotti, Seamus Holden, Florian Steiner and Johannes Hohlbein for actively participating in the open public Twitter ( ) discussion on this topic which can be followed here.

I thank Florian Steiner for sharing the image used for Figure 5 and Gražvydas Lukinavičius for sharing the image and data for Figure 3.

\section{Conflict of interest}

I have no known competing financial interests or personal relationships that could influence the work reported in this paper.

\section{References}

Baer, S. C. (1999). Method and apparatus for improving resolution in scanned optical system. US Patent 5,866,911.

Balzarotti, F., Eilers, Y., Gwosch, K. C., Gynnå, A. H., Westphal, V., Stefani, F. D., Elf, J., and Hell, S. W. (2017). Nanometer resolution imaging and tracking of fluorescent molecules with minimal photon fluxes. Science, 355(6325):606-612.

Betzig, E., Patterson, G. H., Sougrat, R., Lindwasser, O. W., Olenych, S., Bonifacino, J. S., Davidson, M. W., LippincottSchwartz, J., and Hess, H. F. (2006). Imaging intracellular fluorescent proteins at nanometer resolution. Science, 313(5793):1642-1645.

Cnossen, J., Hinsdale, T., Thorsen, R. Ø., Siemons, M., Schueder, F., Jungmann, R., Smith, C. S., Rieger, B., and Stallinga, S. (2020). Localization microscopy at doubled precision with patterned illumination. Nature methods, 17(1):59-63.

Denk, W., Strickler, J. H., and Webb, W. W. (1990). Two-photon laser scanning fluorescence microscopy. Science, 248(4951):7376.

Gall, J. G. (1967). Octagonal nuclear pores. The Journal of cell biology, 32(2):391-399. 
Gerasimaite, R., Bucevicius, J., Kiszka, K. A., Schnorrenberg, S., Kostiuk, G., Koenen, T., and Lukinavicius, G. (2021). Blinking fluorescent probes for tubulin nanoscopy in living and fixed cells. ACS chemical biology.

Gustafsson, M. G. (2000). Surpassing the lateral resolution limit by a factor of two using structured illumination microscopy. Journal of microscopy, 198(2):82-87.

Gwosch, K. C., Pape, J. K., Balzarotti, F., Hoess, P., Ellenberg, J., Ries, J., and Hell, S. W. (2020). Minflux nanoscopy delivers $3 \mathrm{~d}$ multicolor nanometer resolution in cells. Nature methods, $17(2): 217-224$.

Heintzmann, R. (2021). Answers to fundamental questions in superresolution microscopy. Philosophical Transactions of the Royal Society A, 379(2199):20210105.

Heintzmann, R. and Cremer, C. G. (1999). Laterally modulated excitation microscopy: improvement of resolution by using a diffraction grating. In Optical Biopsies and Microscopic Techniques III, volume 3568, pages 185-196. International Society for Optics and Photonics.

Hell, S. W., Lindek, S., Cremer, C., and Stelzer, E. H. (1994). Measurement of the 4pi-confocal point spread function proves $75 \mathrm{~nm}$ axial resolution. Applied Physics Letters, 64(11):13351337.

Hell, S. W. and Wichmann, J. (1994). Breaking the diffraction resolution limit by stimulated emission: stimulated-emissiondepletion fluorescence microscopy. Optics letters, 19(11):780782 .

Hohlbein, J., Diederich, B., Marsikova, B., Reynaud, E. G., Holden, S., Jahr, W., Haase, R., and Prakash, K. (2021). Open microscopy in the life sciences: Quo vadis? arXiv preprint arXiv:2110.13951.

Huang, F., Sirinakis, G., Allgeyer, E. S., Schroeder, L. K., Duim, W. C., Kromann, E. B., Phan, T., Rivera-Molina, F. E., Myers, J. R., Irnov, I., et al. (2016). Ultra-high resolution 3d imaging of whole cells. Cell, 166(4):1028-1040.

Jouchet, P., Cabriel, C., Bourg, N., Bardou, M., Poüs, C., Fort, E., and Lévêque-Fort, S. (2019). Nanometric axial localization of single fluorescent molecules with modulated excitation. BioRxiv, page 865865 .

Li, Y., Mund, M., Hoess, P., Deschamps, J., Matti, U., Nijmeijer, B., Sabinina, V. J., Ellenberg, J., Schoen, I., and Ries, J. (2018). Real-time 3d single-molecule localization using experimental point spread functions. Nature methods, 15(5):367-369.

Lidke, K. A., Rieger, B., Jovin, T. M., and Heintzmann, R. (2005). Superresolution by localization of quantum dots using blinking statistics. Optics express, 13(18):7052-7062.
Löschberger, A., van de Linde, S., Dabauvalle, M.-C., Rieger, B., Heilemann, M., Krohne, G., and Sauer, M. (2012). Superresolution imaging visualizes the eightfold symmetry of gp210 proteins around the nuclear pore complex and resolves the central channel with nanometer resolution. J Cell Sci, 125(3):570575 .

Masullo, L. A., Steiner, F., Zahringer, J., Lopez, L. F., Bohlen, J., Richter, L., Cole, F., Tinnefeld, P., and Stefani, F. D. (2020). Pulsed interleaved minflux. Nano Letters.

Minsky, M. (1988). Memoir on inventing the confocal scanning microscope. Scanning, 10(4):128-138.

Pape, J. K., Stephan, T., Balzarotti, F., Büchner, R., Lange, F., Riedel, D., Jakobs, S., and Hell, S. W. (2020). Multicolor $3 \mathrm{~d}$ minflux nanoscopy of mitochondrial micos proteins. Proceedings of the National Academy of Sciences, 117(34):2060720614.

Prakash, K. (2017). Chromatin architecture: Advances from highresolution single molecule dna imaging. Springer.

Prakash, K. (2021). Laser-free super-resolution microscopy. Philosophical Transactions of the Royal Society A, 379(2199):20200144.

Prakash, K. and Curd, A. (2021). Assessment of 3d minflux data for quantitative structural biology in cells. bioRxiv.

Prakash, K., Diederich, B., Reichelt, S., Heintzmann, R., and Schermelleh, L. (2021). Super-resolution structured illumination microscopy: past, present and future. Philosophical Transactions of the Royal Society A, 379(2199):20200143.

Reymond, L., Huser, T., Ruprecht, V., and Wieser, S. (2020). Modulation-enhanced localization microscopy. Journal of Physics: Photonics, 2(4):041001.

Rossberger, S., Best, G., Baddeley, D., Heintzmann, R., Birk, U., Dithmar, S., and Cremer, C. (2013). Combination of structured illumination and single molecule localization microscopy in one setup. Journal of Optics, 15(9):094003.

Sahl, S. J., Hell, S. W., and Jakobs, S. (2017). Fluorescence nanoscopy in cell biology. Nature reviews Molecular cell biology, 18(11):685.

Schermelleh, L., Ferrand, A., Huser, T., Eggeling, C., Sauer, M., Biehlmaier, O., and Drummen, G. P. (2019). Super-resolution microscopy demystified. Nature cell biology, 21(1):72-84.

Thevathasan, J. V., Kahnwald, M., Cieśliński, K., Hoess, P., Peneti, S. K., Reitberger, M., Heid, D., Kasuba, K. C., Hoerner, S. J., $\mathrm{Li}, \mathrm{Y}$., et al. (2019). Nuclear pores as versatile reference standards for quantitative superresolution microscopy. Nature methods, 16(10):1045-1053. 